\title{
Divide and conquer? Persistence of infectious agents in spatial metapopulations of hosts
}

\author{
Marieke Jesse *, Hans Heesterbeek \\ Theoretical Epidemiology, Faculty of Veterinary Medicine, Utrecht University, the Netherlands
}

\section{A R T I C L E I N F O}

\section{Article history:}

Received 12 July 2010

Received in revised form

17 January 2011

Accepted 20 January 2011

Available online 27 January 2011

Keywords:

Stochastic model

Migration

Movement distance

Patch neighbourhood

Lattice

\begin{abstract}
A B S T R A C T
Persistence of an infectious agent in a population is an important issue in epidemiology. It is assumed that spatially fragmenting a population of hosts increases the probability of persistence of an infectious agent and that movement of hosts between the patches is vital for that. The influence of migration on persistence is however often studied in mean-field models, whereas in reality the actual distance travelled can be limited and influence the movement dynamics. We use a stochastic model, where within- and between-patch dynamics are coupled and movement is modelled explicitly, to show that explicit consideration of movement distance makes the relation between persistence of infectious agents and the metapopulation structure of its hosts less straightforward than previously thought. We show that the probability of persistence is largest at an intermediate movement distance of the host and that spatially fragmenting a population of hosts is not necessarily beneficial for persistence.
\end{abstract}

(c) 2011 Elsevier Ltd. All rights reserved.

\section{Introduction}

Once an infectious disease invades a population, it can go extinct immediately, fade out after an epidemic or persist in the population. In homogeneously mixing populations, infectious agents tend to die out when the host population size is below a critical community size (Bartlett, 1957; Grenfell and Harwood, 1997), related to the demographic processes that regulate the inflow of new susceptible hosts in the population (Anderson and May, 1992). However, populations are often structured in space and then the situation becomes much more subtle. Examples are humans living in cities and towns (Hall et al., 2007), cattle on farms (Le Menach et al., 2005; Kao et al., 2007) or wildlife populations, for example the water voles in the UK (Telfer et al., 2001), the badgers in the UK (Rogers et al., 1998) or the great gerbils in Kazakhstan (Davis et al., 2007a). These populations live in a set of spatially separated patches of suitable habitat, connected to each other by migration of the individuals (e.g. travel of humans between cities, purchasing and selling of cattle and juvenile migration of wildlife). Each patch moreover, has its own dynamics influenced by the incoming and outgoing migrants and by the local conditions. Such a population is called a (spatial) metapopulation (Hanski, 1999; Leibold et al., 2004). The patch size measured is the size of the inhabiting population of interest

\footnotetext{
* Corresponding author. Tel.: +31 30253 1183; fax: +31 302521887

E-mail addresses: m.jesse@uu.nl (M. Jesse),

j.a.p.heesterbeek@uu.nl (H. Heesterbeek).
}

and ranges from very large (e.g. cities, farms) to very small (e.g. wildlife often lives in small (family) groups).

The structure of such metapopulations for a given species is distinct from single homogeneously mixing populations, resulting in differences in dynamics and persistence of the species itself, and other species it interacts with. For example, infectious disease agents both influence and are influenced by the structure and migration of their host (Grenfell and Harwood, 1997). Hanski (1999) showed that, if the total population size is below the critical community size, then spatially fragmenting this population has a positive influence on the ability of an infectious agent to persist and that notably movement of hosts between patches is crucial. The latter aspect has received substantial attention in the literature (e.g. Swinton et al., 1998; Keeling, 2000; Park et al., 2002; Hagenaars et al., 2004; Lindholm and Britton, 2007).

However, there is a much broader range of aspects that influence and control persistence in a metapopulation (Swinton et al., 1998): (i) demographic aspects: birth and death; (ii) epidemic aspects, length of infectious period and the specifics of the transmission process; and (iii) spatial aspects, movement distance of the host and frequency of migration or length of stay in patches. Notably the interaction between these aspects appears to be important, and is not well understood. Indeed, seemingly new phenomena arise from analysis of real systems (Davis et al., 2007b, 2008). In this theoretical paper we aim to contribute to this debate and explore the question: how does the interaction between all the aspects mentioned shape persistence of an infectious disease agent in a spatially structured host population with movement modelled explicitly? 
For studying persistence in a metapopulation often a meanfield approximation is used (Gog et al., 2002; McCallum and Dobson, 2002; Jesse et al., 2008), but here we introduce a (preferred) movement distance of the host: hosts are only allowed to migrate to patches within this movement distance. The set of patches that can be reached by individual hosts migrating out of a given patch within one time step is seen as the neighbourhood of that patch. The effect of the neighbourhood of a patch on persistence has been studied both in an ecological setting (Durrett and Levin, 1994; Hanski and Ovaskainen, 2000; Vuilleumier et al., 2007) and in an epidemiological context (Levin and Durrett, 1996; Rhodes and Anderson, 1996).

Keeling (2000) pointed out that there are two ways of coupling between subpopulations. The first is based on movement rate of individuals between subpopulations, which is the coupling we use in the present paper. The second is by using a parameter that describes how much the global average affects the dynamics of a subpopulation. Keeling used the latter way of coupling and concluded that persistence is maximized at intermediate levels of coupling (in the second interpretation), but expects the qualitative behaviour of both ways of coupling to be similar. In this paper we show that using a movement rate and in addition a movement distance indeed results in persistence being maximized at intermediate levels of coupling.

Here, we take a much broader view by regarding an epidemiological model with demography, explicit modelling of movement (distance and frequency of movement) and coupling of betweenand within-patch dynamics. Among other results, we show that there can be an optimum for persistence at intermediate neighbourhood size. We explore the reasons behind this and how persistence of an infectious agent is influenced by the demographic, epidemic and spatial parameters. Moreover, we show that, in contrast to what was generally believed, a metapopulation is not necessarily more beneficial for the persistence of an infectious agent compared to a single homogeneously mixing population.

\section{Methods}

\subsection{Model}

The model describes a host population divided into subpopulations, each inhabiting a patch with suitable habitat (details in Jesse et al., 2008). In contrast to Jesse et al. (2008) we now consider a spatially explicit metapopulation, where patches are structured in space, in our case on a regular square lattice. At least initially there are no unoccupied patches and the patches are identical in the sense that all parameter values are the same for all subpopulations. An overview of the parameters and their default values used in the numerically studied cases is given in Table 1.

In each patch there is homogeneously mixing and SIR-type dynamics occuring, with hosts being in one of the three following

\section{Table 1}

Definition and default values of the model parameters for numerically studied cases.

\begin{tabular}{lll}
\hline Parameter & Value & Definition \\
\hline$P$ & 100 & Number of patches \\
$K$ & 10 & Carrying capacity \\
$b$ & 0.2 & Birth rate per week \\
$\mu$ & 0.01 & Natural mortality rate per week \\
$m$ & {$[0,1]$} & Movement rate per week \\
$\beta$ & 10 & Transmission rate per week \\
$\gamma$ & 0.5 & Recovery rate per week \\
\hline
\end{tabular}

states: susceptible $(S)$, infectious $(I)$ or recovered/immune to the infection $(R)$. The infectious disease is transmitted directly and hosts do not lose immunity, but eventually die and are replaced by susceptibles. Births are locally density dependent, meaning that they depend on the number of hosts present in that patch.

The model is stochastic and discrete in time, one time step denotes one week, where $S_{x}(t), I_{x}(t)$ and $R_{x}(t)$ denote the number of hosts per infectious state in patch $x$ at time $t$. The total number of hosts in patch $x$ at time $t$ is given by $N_{x}(t)$. Events occur successively in the interval $[t, t+1)$ in the order of birth, death, infection, recovery and migration. Of course, ordering the events is artificial, but it is necessary in a discrete model so that in the bookkeeping individual-level events, such as dying or recovering, are taken into account in a consistent way. The complete set of stochastic difference equations is given by:

$$
\begin{aligned}
& S_{x}(t+1)=S_{x}(t)-\mathbf{D}\left(S_{x}, t\right)-\mathbf{I n f}\left(S_{x}, t\right)+\mathbf{B}\left(N_{x}, t\right)-\mathbf{M}_{\mathrm{out}}\left(S_{x}, t\right)+\mathbf{M}_{\mathrm{in}, S}(x, t) \\
& I_{x}(t+1)=I_{x}(t)-\mathbf{D}\left(I_{x}, t\right)+\mathbf{I n f}\left(S_{x}, t\right)-\mathbf{R e c}\left(I_{x}, t\right)-\mathbf{M}_{\mathrm{out}}\left(I_{x}, t\right)+\mathbf{M}_{\mathrm{in}, I}(x, t) \\
& R_{x}(t+1)=R_{x}(t)-\mathbf{D}\left(R_{x}, t\right)+\operatorname{Rec}\left(I_{x}, t\right)-\mathbf{M}_{\mathrm{out}}\left(R_{x}, t\right)+\mathbf{M}_{\mathrm{in}, R}(x, t)
\end{aligned}
$$

The random variables and their distributions (Table 2) are calculated with the number of hosts at that point in the time interval, using the order of the events; for example, the number of newly infected hosts at time interval $[t, t+1)$ depends on the number of infectious hosts at time $t-1$ minus the number of infectious hosts that died at time $t$.

\subsection{Structure}

Above we described the dynamics within each patch, but these patches are connected to each other via migration of the hosts, which we also model explicitly. Where in Jesse et al. (2008) a mean-field approximation was used, here a finite spatially explicit structure is looked at. The patches to which a host can migrate, are given by the adjacency matrix $A$. This adjacency matrix describes the connections between the patches of the metapopulation: if $A_{i j}=1$ it is possible to move from patch $i$ to patch $j$, otherwise $A_{i j}=0$. We view the metapopulation of patches as being arranged spatially in a finite regular lattice where a node is a patch and where an edge indicates a connection in the above sense. The network is non-directed, which means that if movement is possible from patch $i$ to patch $j$, then it is also possible from patch $j$ to patch $i$, i.e. $A_{i j}=A_{j i}$.

Of particular interest is the movement distance of the hosts, measured as path length in the lattice. For movement distance $d$, a host can migrate along at maximum $d$ edges in the regular lattice of patches. The host can, with equal probability, migrate to all patches that can be reached within this number of edges. This movement distance can be seen as the capacity of the host to migrate to patches at that distance. When the movement distance equals 1 , hosts can only migrate to their nearest neighbours and when the movement distance is large enough, hosts can move to

Table 2

The random variables with their distribution and parameters. The state is given explicitly or by $Z$ with $Z \in\{S, I, R\}$.

\begin{tabular}{llll}
\hline Random variable & Distribution & Parameter & Definition \\
\hline $\mathbf{B}\left(N_{x}, t\right)$ & Poisson & $\max \left[0 ; b N_{x}(t)\left(1-\frac{N_{x}(t)}{K}\right)\right]$ & \# Births \\
$\mathbf{D}\left(Z_{x}, t\right)$ & Binomial & $\mu$ & \# Deaths \\
$\mathbf{I n f}\left(S_{x}, t\right)$ & Binomial & $1-\exp \left(-\frac{\beta I_{x}(t)}{N_{x}(t)}\right)$ & \# Infected \\
& & $\gamma$ & \# Recovered \\
$\mathbf{R e c}\left(I_{x}, t\right)$ & Binomial & $\gamma$ & \# Emigrants \\
$\mathbf{M}_{\text {out }}\left(Z_{x}, t\right)$ & Binomial & $m$ & \# Immigrants \\
$\mathbf{M}_{\text {in }, Z}(x, t)$ & Multinomial & $1 / P$ & \\
\hline
\end{tabular}


every patch. The matrix $M$ gives the patches to which a host can move within a movement distance $d$ and has entries such that, for $i \neq j$ :

$M_{i j}= \begin{cases}1 & \text { if patch } i \text { and } j \text { are connected, i.e. }\left(\sum_{d} A^{d}\right)_{i j}>0 \\ 0 & \text { otherwise }\end{cases}$

and $M_{i i}=0$, preventing hosts from migrating back immediately to the patch they just left (i.e. no loops of length 1 are allowed). The matrix $M$ is calculated by means of $\sum_{d} A^{d}$, where $A_{i j}^{d}$ gives the number of paths of length $d$ from patch $i$ to patch $j$.

\subsection{Simulations}

The model is stochastic and hence there can be large variation between runs of the model, even though the set of parameter values is identical. The stochastic difference equations were simulated for a range of parameter values. The program is written in $\mathrm{R}$ (package 2.10.1, www.cran.r-project.org). At time $t=1$ one infectious host is introduced into one patch in an otherwise completely susceptible metapopulation. For the results the location of this (index) patch, where the first infectious host is introduced, does not matter. This coincides with findings of Pautasso and Jeger (2008), when they studied a model with SI-type dynamics on a directed network, where each node represents an individual.

After introduction of this one infectious host in the metapopulation, there are four possible situations: the infectious agent may (i) not spread at all in the initial patch, (ii) spread in the initial patch, but not between patches (iii) spread within and between patches and then fade out or (iv) spread within and between patches and (quasi)persist in the metapopulation (Jesse et al., 2008). Here, we are interested in the fourth case, persistence in the metapopulation.

We defined the infectious agent to be persistent in a simulation if it is still present in the population after twice the expected life span of the host. The expected life span of a host is $1 / \mu$, therefore the simulations were run for $2 / \mu$ time steps. The choice of twice the expected life span is arbitrary, but within this number of time steps the infectious agent has survived two generations of hosts, and has spread between the patches.

The results obtained are from 1000 simulations. This number of simulations per datapoint was chosen such that variation between several runs of 1000 simulations for the same set of parameter values is small. Therefore, increasing the number of simulations smoothens the graphs depicting our results, but does not alter or add to conclusions we draw.

The default parameter values are shown in Table 1 . The default death rate equals 0.01 , meaning that the life span of a host is on average 100 weeks, i.e. a host lives around two years. And the default recovery rate equals 0.5 , so that a host is infectious for on average two weeks. The metapopulation consists of 100 patches, all with carrying capacity 10 . This carrying capacity is chosen such that persistence of the infectious agent in a single patch is not possible. In a single homogeneously mixing population of size 1500 around $90 \%$ of the simulations will result in a persisting infection for the default parameter values. The metapopulation is arranged on a square $10 \times 10$ lattice, with reflective boundaries. These boundaries can be considered as natural boundaries such as water or mountains, which cannot be crossed by the host.

\section{Results and conclusions}

In this section we will first present the influence of the spatial aspects on persistence of infectious agents in the metapopulation.
Then the demographical and epidemiological aspects will be presented.

\subsection{Spatial aspect}

In Fig. 1a, the influence of the spatial aspects on persistence of the infectious agent are explored. In this figure, contour lines show the fractions of simulations with persisting infection as functions of the migration rate and the movement distance of the host. Fixing the movement distance and then increasing the migration rate resulted in a higher fraction of simulations with

a

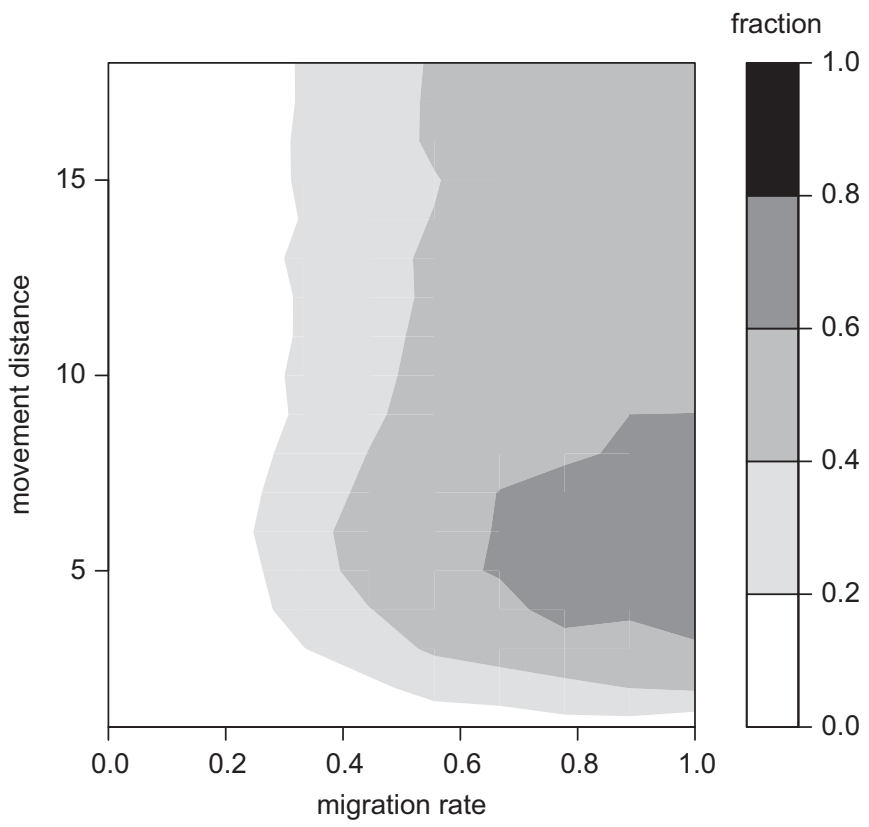

b

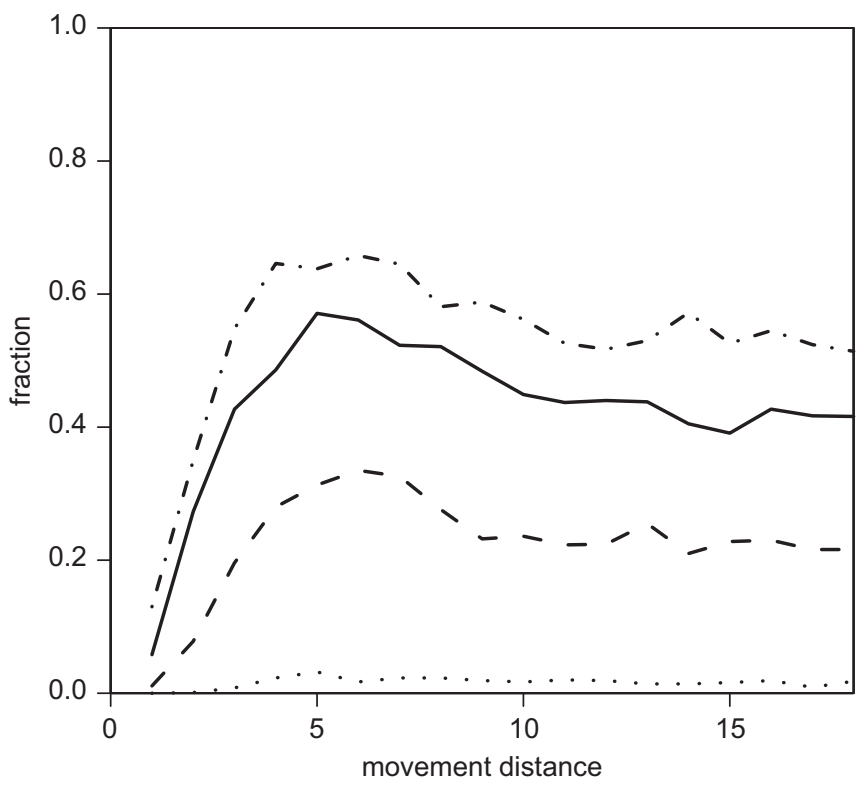

Fig. 1. In (a) the contour lines mark the area where the combination of the migration rate and the movement distance yield the same minimum fraction of simulations with persisting infection. In (b) the fraction of simulations with persisting infection is shown with migration rate 0.2 (dotted line), 0.4 (dashed line), 0.6 (solid line) and 0.8 (dot-dashed line). The results are taken from 1000 simulations and the default parameter values are $P=100, K=10, \beta=10, \gamma=0.5$ $b=0.2$ and $\mu=0.01$. 
persisting infection. A higher migration rate means that hosts spend a shorter time in one patch and thus change patches more frequently. By changing patches more frequently, hosts increase the number of contacts with other hosts and therefore have a higher probability of finding sufficient susceptible hosts to infect while they are still infectious.

Fixing the migration rate and increasing the movement distance shows a maximum in the fraction of simulations with persisting infection at intermediate movement distance. This result is also shown in Fig. 1b for four different migration rates. Clearly there is a peak at intermediate movement distance, except for relatively low migration rates (a migration rate of 0.2 means that a host moves once every five weeks). The default infectious period is two weeks, so at low migration rates the host spends, on average, most of its entire infectious period within one patch. Since the patch size is too low for persistence, there is only a very small fraction of simulations showing persistence.

\subsection{Effect of movement distance on patch level}

As already shown, varying only the movement distance already results in dissimilarities between the fraction of simulations with persisting infection. In order to understand where these dissimilarities come from, we focus on the differences that occur on patch level. Because the infectious hosts are the key for persistence of the infectious agent in the metapopulation, we focused on some properties of patches with respect to infectious hosts that might be influenced by the movement distance:

(i) the total number of time steps (not necessarily consecutive) that a patch contains at least one infectious host;

(ii) the number of times that a patch gets infected, i.e. how often are there transitions from no infectious host in a patch to at least one infectious host in that patch;

(iii) the total number of infectious hosts per patch, i.e. the sum of the number of infectious hosts at each time step in a patch;

(iv) the number of consecutive time steps a patch contains an infectious host.

Only simulations with persisting infection were taken into account when calculating the above measures. The results are presented as box-and-whisker-plots in Fig. 2.

It is immediately clear that the medians for movement distance 6 and 14 are approximately the same for all four measures, but that the range differs: the variation between patches for intermediate movement distance is larger than for large movement distance. Movement distance 14 corresponds to a situation where the patches are almost fully connected and the system starts behaving like one single homogeneously mixing population.

On average a patch contains 35 out of the 200 time steps (17.5\% of the time) at least one infectious host for low movement distance (Fig. 2a). The average number for the other movement a

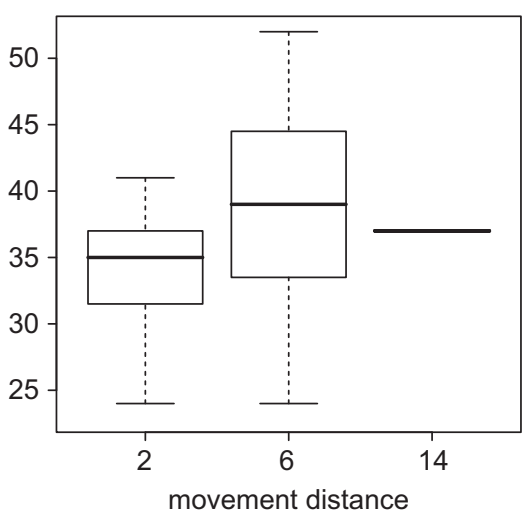

C

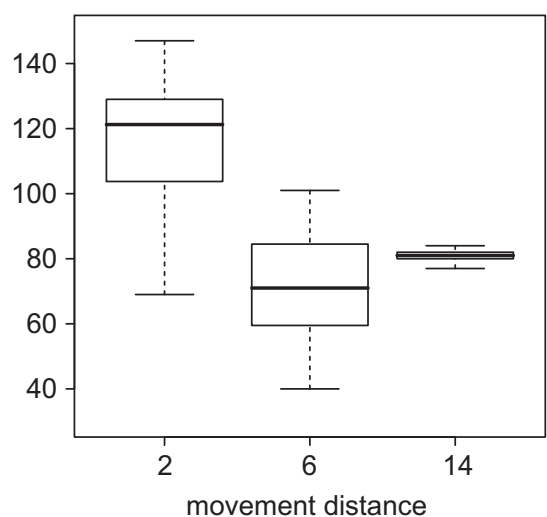

b

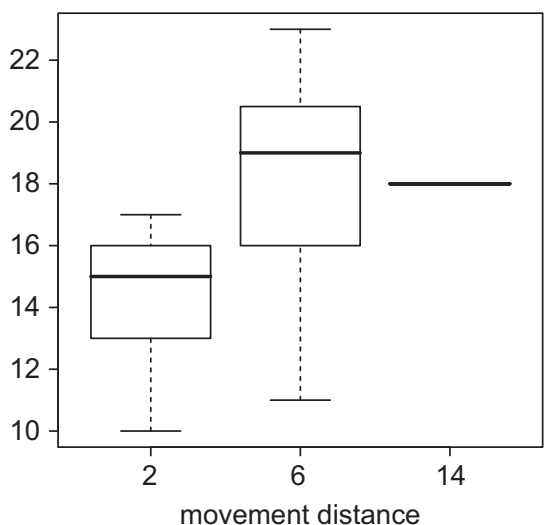

d

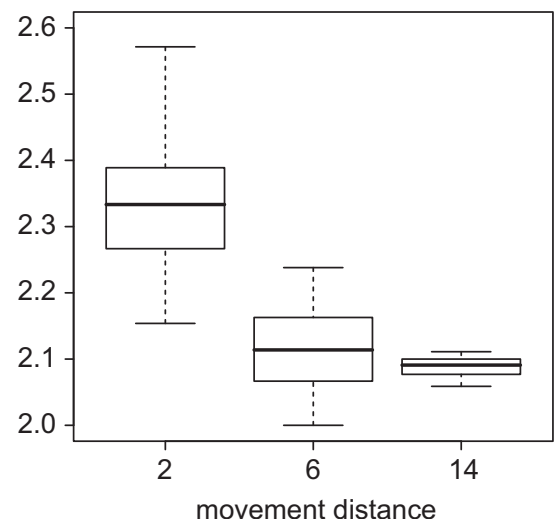

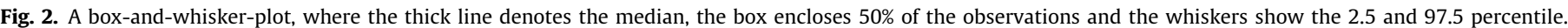

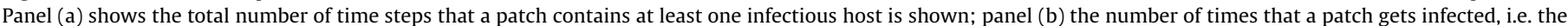

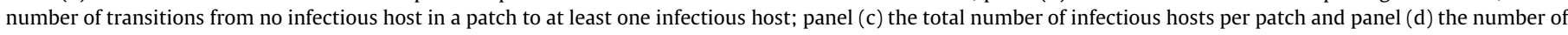

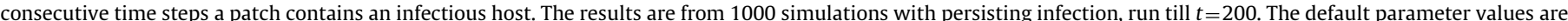
$P=100, K=10, \beta=10, \gamma=0.5, b=0.2$ and $\mu=0.01$. 
distances is slightly higher. The number of transitions from no infectious hosts in a patch to at least one is the lowest for the low movement distance (median 15 times, Fig. 2b). This might be due to (infectious) hosts returning more often back to a patch they visited just a few time steps ago (fewer patches to choose from). On average the total number of infectious hosts per patch is about 1.5 times larger for low movement distance (median of 120) than for the other two movement distances (Fig. 2c). Because the number of infected patches at each time step is equal for all movement distances, the number of infectious hosts in each patch must be higher for low movement distance than for the other movement distances. Again, this is probably caused by hosts returning more often to a patch they just visited at low maximum movement distances. Finally in Fig. $2 d$ the median number of consecutive time steps a patch is inhabited by infectious hosts is shown. Basically, this is just the total number of time steps a patch is infected divided by the number of transitions from having no infectious host in a patch to having at least one. Here, the low movement distance shows that a patch contains at least one infectious host for a longer uninterrupted number of time steps.

With the four measures we looked at, we can conclude that at low movement distance, patches are on average longer infected and contain over the whole time period more infectious hosts than at large movement distance. This implies that the infectious agent remains within the same area at low movement distance until that area runs too low on available susceptible hosts. At large movement distance on the other hand, the infectious agent is more scattered throughout the whole metapopulation, patches get (re)infected more often, but it is for a short period of time and with only a few infectious hosts. Hence, at low movement distance the spreading of the infectious agent occurs more locally and gradually becomes more global when the movement distance increases.

\subsection{Demographical and epidemiological aspects}

As mentioned earlier, persistence of an infectious agent is also influenced by demographical and epidemiological aspects. The fraction of simulations with persisting infection is therefore studied as function of each of the four parameters controlling these aspects: birth rate, death rate, transmission rate and recovery rate. The fraction is shown in Fig. 3 for three different movement distances, namely 2, 6 and 14, and for a single homogeneously mixing population.

This single homogeneously mixing population consists of one patch with a carrying capacity $K$ of 1000 hosts; the total size of the metapopulation and the single population are therefore equal. Moreover, this figure shows how a change in a relevant parameter influences the fraction of simulations with persisting infection. For all parameters, the fraction of simulations with persisting infection is the highest for intermediate movement distance, corresponding to the previous result that there is a maximum in the fraction of simulations with persisting infection at this movement distance.

The natural death rate (i.e. not related to the infection) determines the life span of a host and influences also, because of the density-dependent birth rate, the population turnover: a lower death rate implies a longer life span and therefore a slower a

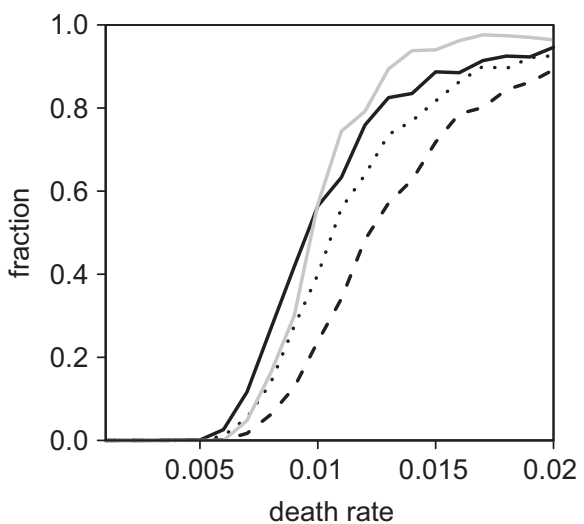

C

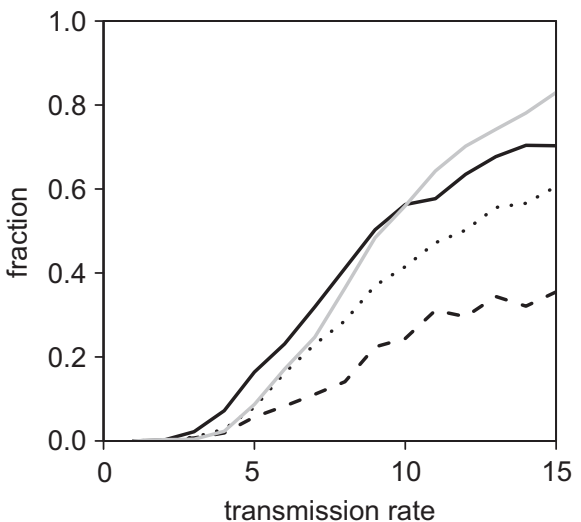

b

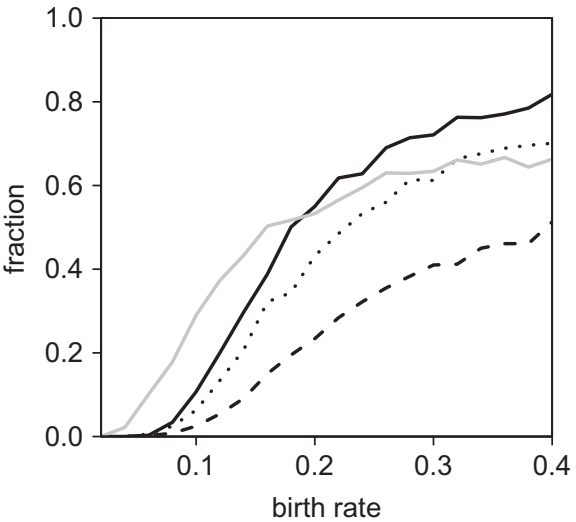

d

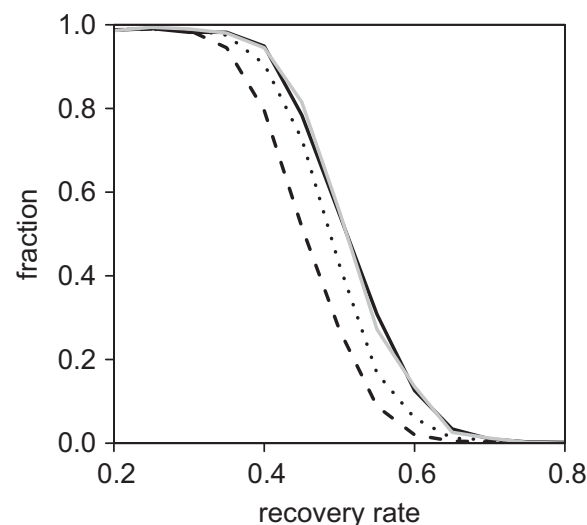

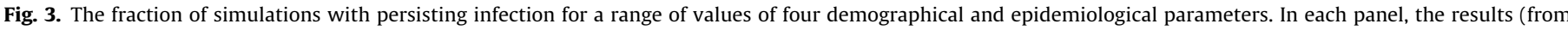

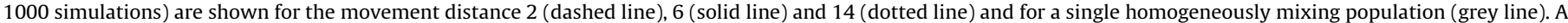
migration rate of 0.6 is used, and the default parameter values are $P=100, K=10, \beta=10, \gamma=0.5, b=0.2$ and $\mu=0.01$. 
population turnover; i.e. a slower inflow of new susceptible hosts. The life span is used to define when an infectious agent is persistent in our set-up, so varying the death rate means that the number of time steps before an infectious agent is called persistent varies as well. Increasing the death rate, i.e. increasing population turnover, has a positive effect on the fraction of simulations with persisting infection (Fig. 3a). At a low death rate, while the speed by which the infectious agent spreads through the whole population remains equal, it takes longer for new susceptible hosts to arise. This time gap is more difficult to bridge in a single homogeneously mixing population, where all susceptible hosts are immediately available for contact to the infectious agent. Therefore, a low death rate is more beneficial for persistence in a metapopulation than in a single homogeneously mixing population.

The birth rate in this model is density dependent; a high birth rate implies that each subpopulation is around carrying capacity and dead hosts are replaced by susceptible new-borns quickly. Hence, increasing the birth rate also increases the fraction of simulations with persisting infection (Fig. 3b). At low birth rate, a single homogeneously mixing population is more beneficial for the infectious agent, because the few susceptible hosts that are born per unit of time are found quickly, while in the metapopulation an infectious host needs time to find the susceptible hosts left. When the birth rate is large, susceptible hosts arise faster and are immediately available for contact with infectious hosts in case of a single homogeneously mixing population. This could mean that the infectious agent 'burns through' the available susceptibles too fast to allow persistence. It is then advantageous for the infectious agent to be in the metapopulation: not all susceptible hosts are immediately available for contact, allowing the infectious agent to persist in the population for a longer time (i.e. the risk of fade out is spread).

In Fig. 3c the fraction of simulations with persisting infection is shown for various transmission rates. For low transmission rates the fraction of simulations with persisting infection is higher in a metapopulation than in a single homogeneously mixing population, but the opposite holds at high transmission rates. In a single homogeneously mixing population an infectious host has immediate access to all susceptible hosts. At high transmission rate, this availability of all susceptibles in a single homogeneously mixing population is beneficial for the infectious agent, because even when there are only a few susceptibles left, there is a high probability that they will be contacted. In a metapopulation on the other hand, an infectious host needs time to travel to patches where the few susceptibles remain, and before successful contact has been made this infectious host may have died or recovered. At low transmission rate, the above situations are reversed.

The recovery rate determines the infectious period of a host and Fig. $3 d$ shows that the fraction of simulations with persisting infection as a function of recovery rate is hardly influenced by the movement distance of the host. When the recovery rate is large, the infectious period is short and, on average, fewer infectious hosts leave the patch during the infectious period, making it more difficult for the infectious agent to spread to other patches. The movement distance does not have a large influence on this, because the ability to spread is here, in the first instance, mostly determined by the chance to leave the patch at all.

Furthermore, a short infectious period results in a population of many recovered hosts. In Fig. 3d, the fraction of simulations with persisting infection coincides for both the single homogeneously mixing population and a metapopulation where hosts have an intermediate movement distance. However, this fraction is very much influenced by the migration rate of the host (not shown). Persistence of the infectious agent is more difficult at low migration rate, because the infectious host does not change patches frequently enough to sustain the infectious agent. A high migration rate in a metapopulation performs slightly better than a single homogeneously mixing population, because running out of susceptible hosts happens faster in a single population than in a metapopulation.

Finally, both the shape of the lattice and the number of patches do not qualitatively change the results (not shown). Reshaping the lattice, for example into $4 \times 25$ with the same total population size as the $10 \times 10$ case, still gives a peak at the same intermediate movement distance of 6 . Interestingly, the mean distance of one patch to any other patch is for the $4 \times 25$ lattice 9.2 steps and for the $10 \times 10$ lattice 6.6. Increasing the number of patches to, for example, a $12 \times 12$ lattice also gives a peak at intermediate movement distance. The latter peak occurs at a slightly higher movement distance compared to the above cases, however, the $12 \times 12$ case is not directly comparable, because both the total population size and the mean distance from one patch to any other is larger. Thus the size of the lattice does not appear to essentially change the results.

\subsection{Balance}

Persistence of an infectious agent in a metapopulation results from a balance between the spatial, demographical and epidemiological components. We studied the components in isolation above, but now look at their combined effect (Fig. 4).

Each panel in this figure shows curves leading to the same fraction of simulations with persisting infection (isopersistence level') as function of the death and the transmission rate. The isopersistence levels are shown for low, intermediate and high movement distance in a metapopulation and for a single homogeneously mixing population and for the four parameter combinations low/high migration rate and low/high birth rate. For all four subfigures the default recovery rate is used, because Fig. 3d showed that for the various movement distances differences in the fraction of simulations with persisting infection are not strongly affected by the recovery rate. In order to compare situations we used in Fig. 4a, c an isopersistence level of 0.3 and in Fig. 4b, $d$ a level of 0.6. These levels are chosen with use of Fig. 3d, where for migration rate 0.4 the fraction of simulations with persisting infection is around 0.3 at the peak and for migration rate 0.8 around 0.6 .

At low birth rate the total population size is smaller than at high birth rate, because in the latter situation the population is close to carrying capacity. For low birth rates both the panels with low and high migration rates (Fig. 4a and b) show that in a single homogeneously mixing population the isopersistence level is achieved at much lower transmission and death rates than in a metapopulation. In that situation, a single homogeneously mixing population is thus more beneficial for the infectious agent than a metapopulation, because at a low birth rate susceptible hosts are not replenished at the same rate as at a high birth rate; it takes more time before new susceptible hosts arise. When they do arise it is more difficult for an infectious host in a metapopulation to successfully meet this susceptible host, because the infectious host first has to move to a patch with sufficient susceptible individuals within its infectious period. In a single homogeneously mixing population the new susceptible hosts are immediately available for contact.

At high birth and low migration rate (Fig. 4c), the isopersistence level for the single homogeneously mixing population and the intermediate movement distance almost coincide. At high transmission rate the isopersistence levels of all situations coincide, because infectious hosts then have successful contacts with more susceptible hosts per unit of time. 

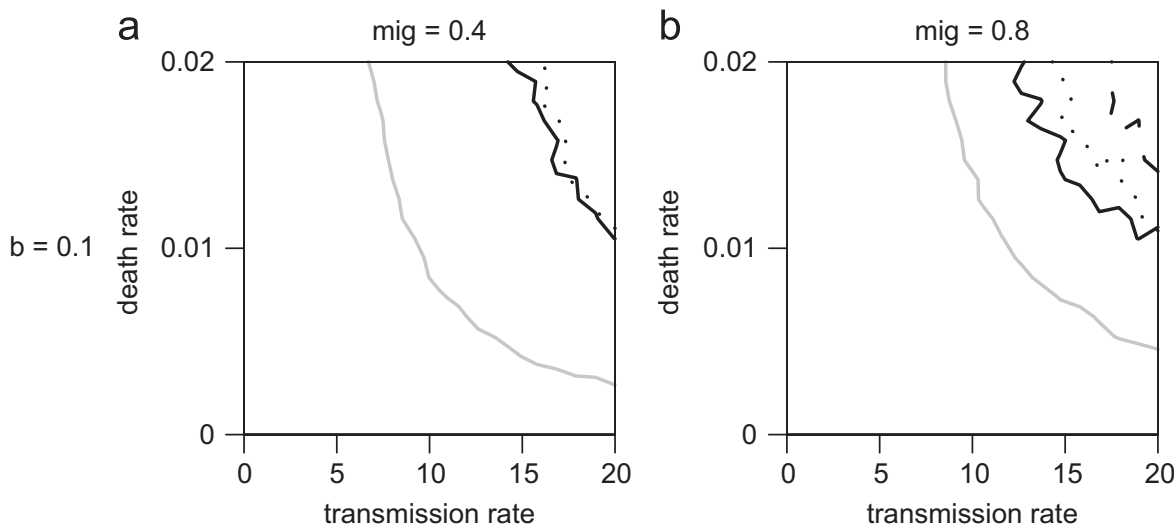

C

d
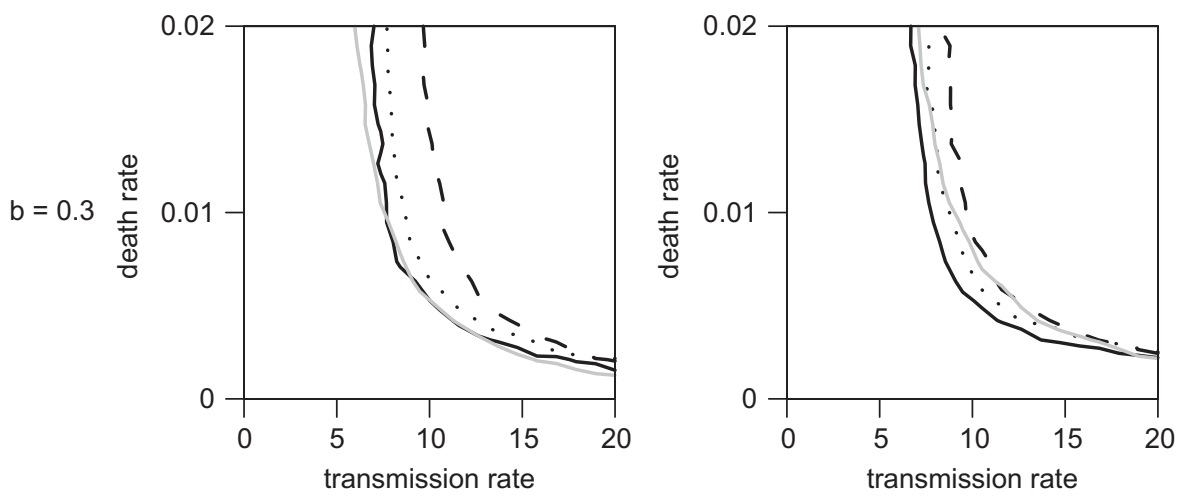

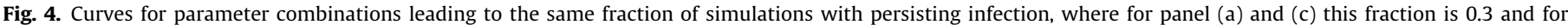

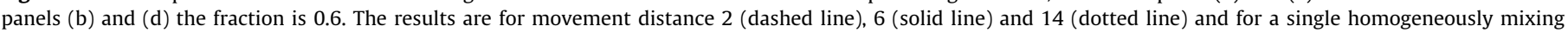

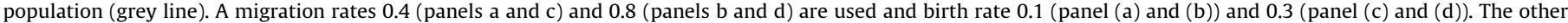
parameters are set to the default values: $P=100, K=10$ and $\gamma=0.5$.

At both high birth and high migration rate (Fig. 4d), the differences between the four cases are very small, especially at a large transmission rate all movement distances coincide more or less with the single homogeneous mixing population.

All panels in this figure show the same pattern as described in previous sections: persistence of an infectious agent in a metapopulation is easier accomplished at intermediate movement distance of the host.

\section{Discussion}

In this paper we showed that persistence of an infectious agent in a metapopulation results from of a balance between spatial, demographical and epidemiological parameters. We showed on lattices a robust non-linear relation between the fraction of simulations with persisting infection and movement distance of hosts, with this fraction being largest at an intermediate movement distance of the host. Furthermore we showed that dividing a single homogeneously mixing population into several smaller populations that are connected to each other, is not necessarily beneficial for the persistence of the infectious agent.

The insight we obtain is based on simulations only. While an important part of relevant parameter space has been explored, there is considerable additional value in providing analytical proof of the observed qualitative phenomena, to obtain robustness of results and deeper understanding. There have been many approaches in the literature where authors have focussed on analytical results concerning infectious disease in spatial metapopulations, and even more concerning single-species populations, predator-prey systems, and notably host-parasitoid systems. Mostly these studies concentrate on stability analysis of the trivial equilibrium (i.e. invasion problems, see Jansen and Lloyd, 2000 and references given there) or on providing analytical expressions for the expected time to extinction from a quasi-steady state (see Nåsell, 1999; Hagenaars et al., 2004; Lindholm and Britton, 2007 and the references given there), as persistence in stochastic models is always temporary (Hanski, 1998; Lloyd-Smith et al., 2005).

In discrete-time models it is not possible to determine the quasi-stationary distribution and other measures have to be used; for example, persistence time (Rhodes and Anderson, 1996) or a certain number of time steps the infectious disease is present in the metapopulation (Swinton et al., 1998; Vuilleumier et al., 2007; Jesse et al., 2008; Courcoul and Ezanno, 2010). We focus on persistence (long-term quasi-stability), i.e. we look at the fraction of realisations of the stochastic process persisting for a time beyond a certain minimum (set arbitrarily by twice the average life span of the host species). We noticed that increasing this minimum did not qualitatively change the results. However, taking twice the life span of the host as measure for persistence for hosts with very short life spans, may lead to a time scale that is too short to speak of persistence.

In our system we merged a stochastic description with discrete spatial structure, with on top of that non-uniform dispersal (due to a maximum movement distance for hosts). This combination did not allow us to obtain analytical results, nor does the set-up allow for easy simplified models with only two patches, say, that might be amenable to direct analysis. We can, however, compare to studies where discrete space was studied 
analytically with different choices for dispersal. Rohani et al. (1996) conclude that, in predator-prey type interaction with uniform dispersal, the equilibrium becomes unstable, compared to a single homogeneous population, when there is a big difference in the dispersal between the two species. We have identical dispersal for our two 'predator-prey species' (infected and susceptible individuals, respectively). In our set-up we show, by simulation, differences in persistence, if dispersal is not uniform, but constrained. In the discussion of Rohani et al. (1996), it is argued that the equilibrium may destabilise when dispersal is not uniform. Similar equilibrium (in)stability results are discussed analytically by White and White (2005) studying coupled map lattices with integro-differential equations. They show in addition that destabilising influences of dispersal can only occur in 'exploiter-victim' type of relationships.

In a much simpler model, Funk et al. (2005) study the dynamics of virus particles in a spatial metapopulation of host cells. This is basically a one-species system because only the virus particles disperse. The dispersal mechanism, however, is closer to ours, as dispersal is constrained to be to (eight) nearest neighbours only. The analytical results allow a large class of dispersal mechanisms to be studied. One of their results is that if dispersal is too fast the infection cannot be maintained. Webb et al. (2007) show, for a host-parasite system, that highly local systems, i.e. where dispersal is limited, have reduced persistence of the infectious agent. Both these observations (Funk et al., 2005; Webb et al., 2007) are, although from very different models, in broad agreement with our finding of an optimal persistence for intermediate dispersal frequency and distance. This is in contrast to results by Hagenaars et al. (2004) who study persistence (measured as time to extinction from a quasi steady state and as mean outbreak duration) in a spatial and stochastic SIR set-up similar to ours. Their analytical and simulation results show a rather more monotone rise in persistence as a function of increasing between-patch contacts. However, there is an important difference with our model: their model has no actual dispersal within the metapopulation, the only migration comes from outside the system, and is essentially a model with two levels of contacts (within-patch and with individuals in other patches) for individuals that are otherwise fixed in their patch. Together, the above comparisons to related studies lead us to expect that our observation of non-monotone persistence as a function of coupling crucially depends on the physical migration between patches of individuals of at least two types in an 'exploiter-victim' relationship, with constraints on the distance over which individuals can migrate within a time step.

An important characteristic of a metapopulation is that the infectious hosts have to move to find susceptible hosts. At low movement distance infectious hosts encounter a lower number of susceptible hosts than the average host, because the number of susceptible hosts is depleted by the presence of other infectious hosts. These infectious hosts are competing with each other over the available susceptibles and thereby reduce the potential for each one of them to transmit the infectious disease to a susceptible (Rhodes and Anderson, 2008). At a relatively high migration rate, i.e. with a short sojourn time per patch, a host can meet more others than at a low migration rate. An infectious host will therefore have a larger number of contacts with susceptible hosts, because they visit more patches during their infectious period. Compared to a single homogeneously mixing population, hosts in metapopulations can have the advantage that they do not reach all susceptible hosts at the same time, which can improve the probability of survival of the infectious agent.

As is also shown by Cross et al. (2005), the relation between migration and recovery is very important for the ability of an infectious agent to persist, because the infectious period determines the time an infectious host has to infect susceptible hosts. And the migration rate is an important determinant of the level of mixing.

Regarding movement we studied a wide range of migration rates: from migrating almost never, to migrating every week. We also assumed that all individuals experience the same migration rate. In reality there is a lot of difference in movement behaviour among species (Hawkes, 2009). Movement may, for example, be restricted to mainly juveniles leaving their nest to settle elsewhere or to foraging outside the own habitat patch. The first mechanism can be thought of at low migration rates, although there are no age-classes defined in the model. The latter one at high migration rates, where individuals leave their own patch, visit another one and then return to their home patch again.

This situation could be even more resembled when the probability to move to a certain patch depends on the distance of that patch with respect to the resident patch. We assumed random dispersal, within the movement distance, but it can also be argued that hosts may have a preference for nearby patches. The influence of a dispersal function, favouring nearby patches, on the persistence of the infectious agent should therefore be studied more carefully.

In this paper we defined the movement distance by the number of edges crossed. Another option would have been using a radial movement distance, where individuals can move to all patches within a certain radius around their resident patch. However, using a radial movement distance instead, does not affect the results. Durrett and Levin (1994) also concluded that the qualitative behaviour is independent of the exact definition of movement distance when they studied interacting particle systems for various definitions of movement distance.

There have been studies of the effect of the neighbourhood of a patch on the persistence of the infectious agent in a spatially structured population. In an ecological context Vuilleumier et al. (2007) and in an epidemiological context Rhodes and Anderson (1996) concluded that increasing the movement distance maximises the probability of persistence. They considered patches as the unit of study and had many unoccupied patches in the population. Another study (Courcoul and Ezanno, 2010) did take within-patch dynamics into account, but they also considered indirect transmission. The probability of infection of a susceptible host in a patch depended not only on the available infectious hosts in the same patch, but also on the prevalence of the infection in neighbouring patches. Courcoul and Ezanno (2010) varied the number of neighbouring patches that influence the infection dynamics in a patch, but did not see a peak in the fraction of simulations with persisting infection at an intermediate number of neighbours. However, due to the different ways persistence can be defined, one should be cautious in comparing the various studies.

The model used in this paper is as simple as possible. For example, it is not taken into account that infectious hosts might experience reduced mobility or death as a result of the infectious disease. Another assumption is continuous birth, as opposed to seasonal birth, where births occurs in a short period of a year, which is often the case in animal populations. Seasonal births reduce the probability of persistence of the infectious agent, because there are fewer infectious hosts between epidemics, which increases the probability of fade-out due to stochasticity (Grassly and Fraser, 2006). But, each year there is also a burst of new susceptible hosts into the population (Altizer et al., 2006), creating the possibility of a new epidemic. One of the next steps in exploring the balance of the various aspects (spatial, demographical and epidemiological) involved in the persistence of an infectious agent would be to include one or more of the abovementioned options. 


\section{Acknowledgement}

This research is supported by The Netherlands Organisation for Scientific Research (NWO grant 918.56.620).

\section{References}

Altizer, S., Dobson, A., Hosseini, P., Hudson, P., Pascual, M., Rohani, P., 2006. Seasonality and the dynamics of infectious diseases. Ecol. Lett. 9, 467-484.

Anderson, R.M., May, R.M., 1992. Infectious Diseases of Humans: Dynamics and Control. Oxford University Press, USA.

Bartlett, M.S., 1957. Measles periodicity and community size. J. R. Stat. Soc. Ser. A Stat. Soc. $120,48-70$.

Courcoul, A., Ezanno, P., 2010. Modelling the spread of bovine viral diarrhoea virus (bvdv) in a managed metapopulation of cattle herds. Vet. Microbiol. 142, 119-128.

Cross, P.C., Lloyd-Smith, J.O., Johnson, P.L.F., Getz, W.M., 2005. Duelling timescales of host movement and disease recovery determine invasion of disease in structured populations. Ecol. Lett. 8, 587-595.

Davis, S., Klassovskiy, N., Ageyev, V., Suleimenov, B., Atshabar, B., Klassovskaya, A., Bennett, M., Leirs, H., Begon, M., 2007a. Plague metapopulation dynamics in a natural reservoir: the burrow system as the unit of study. Epidemiol. Infect. $135,740-748$.

Davis, S., Leirs, H., Viljugrein, H., Stenseth, N.C., Bruyn, L.D., Klassovskiy, N., Ageyev, V., Begon, M., 2007b. Empirical assessment of a threshold model for sylvatic plague. J. R. Soc. Interface 4, 649-657.

Davis, S., Trapman, P., Leirs, H., Begon, M., Heesterbeek, J.A.P., 2008. The abundance threshold for plague as a critical percolation phenomenon. Nature 454, 634-637.

Durrett, R., Levin, S.A., 1994. Stochastic spatial models: a user's guide to ecological applications. Philos. Trans. R. Soc. B 343, 329-350.

Funk, G.A., Jansen, V.A.A., Bonhoeffer, S., Killingback, T., 2005. Spatial models of virus-immune dynamics. J. Theor. Biol. 233, 221-236.

Gog, J., Woodroffe, R., Swinton, J., 2002. Disease in endangered metapopulations: the importance of alternative hosts. Proc. R. Soc. B 269, 671-676.

Grassly, N.C., Fraser, C., 2006. Seasonal infectious disease epidemiology. Proc. R. Soc. B 273, 2541-2550.

Grenfell, B., Harwood, J., 1997. (Meta)population dynamics of infectious diseases. Trends Ecol. Evol. 12, 395-399.

Hagenaars, T.J., Donnelly, C.A., Ferguson, N.M., 2004. Spatial heterogeneity and the persistence of infectious diseases. J. Theor. Biol. 229, 349-359.

Hall, I.M., Egan, J., Barrass, I., Gani, R., Leach, S., 2007. Comparison of smallpox outbreak control strategies using a spatial metapopulation model. Epidemiol. Infect. 135, 1133-1144.

Hanski, I., 1998. Metapopulation dynamics. Nature 396, 41-49.

Hanski, I., 1999. Metapopulation Ecology. Oxford University Press.

Hanski, I., Ovaskainen, O., 2000. The metapopulation capacity of a fragmented landscape. Nature 404, 755-758.

Hawkes, C., 2009. Linking movement behaviour, dispersal and population processes: is individual variation a key? J. Anim. Ecol. 78, 894-906.

Jansen, V.A., Lloyd, A.L., 2000. Local stability analysis of spatially homogeneous solutions of multi-patch systems. J. Math. Biol. 41 (3), 232-252.
Jesse, M., Ezanno, P., Davis, S., Heesterbeek, J.A.P., 2008. A fully coupled, mechanistic model for infectious disease dynamics in a metapopulation: movement and epidemic duration. J. Theor. Biol. 254, 331-338.

Kao, R.R., Green, D.M., Johnson, J., Kiss, I.Z., 2007. Disease dynamics over very different time-scales: foot-and-mouth disease and scrapie on the network of livestock movements in the uk. J. R. Soc. Interface 4, 907-916.

Keeling, M.J., 2000. Metapopulation moments: coupling, stochasticity and persistence. J. Anim. Ecol. 69, 725-736.

Le Menach, A., Legrand, J., Grais, R.F, Viboud, C, Valleron, A, Flahault, A., 2005. Modeling spatial and temporal transmission of foot-and-mouth disease in france: identification of high-risk areas. Vet. Res. 36, 699-712.

Leibold, M.A., Holyoak, M., Mouquet, N., Amarasekare, P., Chase, J.M., Hoopes, M.F. Holt, R.D., Shurin, J.B., Law, R., Tilman, D., Loreau, M., Gonzalez, A., 2004. The metacommunity concept: a framework for multi-scale community ecology. Ecol. Lett. 7, 601-613.

Levin, S.A., Durrett, R., 1996. From individuals to epidemics. Philos. Trans. R. Soc. B 351, 1615-1621.

Lindholm, M., Britton, T., 2007. Endemic persistence or disease extinction: the effect of separation into sub-communities. Theor. Popul. Biol. 72, 253-263.

Lloyd-Smith, J.O., Cross, P.C., Briggs, C.J., Daugherty, M., Getz, W.M., Latto, J. Sanchez, M.S., Smith, A.B., Swei, A., 2005. Should we expect population thresholds for wildlife disease? Trends Ecol. Evol. 20, 511-519.

McCallum, H., Dobson, A., 2002. Disease, habitat fragmentation and conservation. Proc. R. Soc. B 269, 2041-2049.

Nåsell, I., 1999. On the time to extinction in recurrent epidemics. J. R. Stat. Soc. Ser B. Stat. Methodol. 61, 309-330.

Park, A.W., Gubbins, S., Gilligan, C.A., 2002. Extinction times for closed epidemics: the effects of host spatial structure. Ecol. Lett. 5, 747-755.

Pautasso, M., Jeger, M.J., 2008. Epidemic threshold and network structure: the interplay of probability of transmission and of persistence in small-size directed networks. Ecol. Complex. 5, 1-8.

Rhodes, C., Anderson, R., 1996. Persistence and dynamics in lattice models of epidemic spread. J. Theor. Biol. 180, 125-133.

Rhodes, C.J., Anderson, R.M., 2008. Contact rate calculation for a basic epidemic model. Math. Biosci. 216, 56-62.

Rogers, L., Delahay, R., Cheeseman, C., Langton, S., Smith, G.C. Clifton-Hadley, R.S 1998. Movement of badgers (meles meles) in a high-density population: individual, population and disease effects. Proc. R. Soc. B 265, 1269-1276.

Rohani, P., May, R.M., Hassell, M.P., 1996. Metapopulations and equilibrium stability: the effects of spatial structure. J. Theor. Biol. 181, 97-109.

Swinton, J., Harwood, J., Grenfell, B.T., Gilligan, C.A., 1998. Persistence thresholds for phocine distemper virus infection in harbour seal phoca vitulina metapopulations. J. Anim. Ecol. 67, 54-68.

Telfer, S., Holt, A., Donaldson, R., Lambin, X., 2001. Metapopulation processes and persistence in remnant water vole populations. Oikos 95, 31-42.

Vuilleumier, S., Wilcox, C., Cairns, B.J., Possingham, H.P., 2007. How patch configuration affects the impact of disturbances on metapopulation persistence. Theor. Popul. Biol. 72, 77-85.

Webb, S.D., Keeling, M.J., Boots, M., 2007. Host-parasite interactions between the local and the mean-field: how and when does spatial population structure matter? J. Theor. Biol. 249, 140-152.

White, S.M., White, K.A.J., 2005. Relating coupled map lattices to integro-difference equations: dispersal-driven instabilities in coupled map lattices. J. Theor. Biol. 235, 463-475. 